\title{
P3HT:PCBM Bulk-Heterojunctions: Observing Interfacial and Charge Transfer States with Surface Photovoltage Spectroscopy
}

Frank E. Osterloh, ${ }^{* \dagger}$ Michael A. Holmes, ${ }^{\dagger}$ Jing Zhao, ${ }^{\dagger}$ Lilian Chang, ${ }^{\ddagger}$ Steven Kawula, ${ }^{\ddagger}$ John D. Roehling, and Adam J. Moulé

${ }^{\dagger}$ Department of Chemistry, and Department of Chemical Engineering and Materials Science, University of California, One Shields Avenue, Davis, California 95616, United States

Supporting Information

ABSTRACT: Surface photovoltage (SPV) spectra are reported for separate films of $(6,6)$-phenyl- $\mathrm{C}_{61}$-butyric acid methyl ester (PCBM) and for regioregular and regiorandom poly(3-hexylthiophene) (P3HT):PCBM bulk heterojunctions, as a function of wavelength, film thickness, thermal annealing, and substrate. In PCBM films, two photovoltage features are observed at 1.1-1.4 eV (F1) and 1.4-2.3 eV (F2), which are assigned to excitation of charge transfer states at the interface (F1) and in the bulk (F2) of the film. In BHJ films, five different photovoltage features are observed at $0.75-0.9 \mathrm{eV}$ (F1), 0.9-1.3 eV (F2), 1.3-1.8 eV (F3), 1.8-2.0 eV (F4), and 2.0-2.4 eV (F5). This data can be analyzed on the basis of optical absorbance and fluorescence spectra of the films, and using SPV spectra for PCBM and P3HT only films, and for a BHJ film containing P3HT nanofibers for comparison. SPV features are assigned to states at the

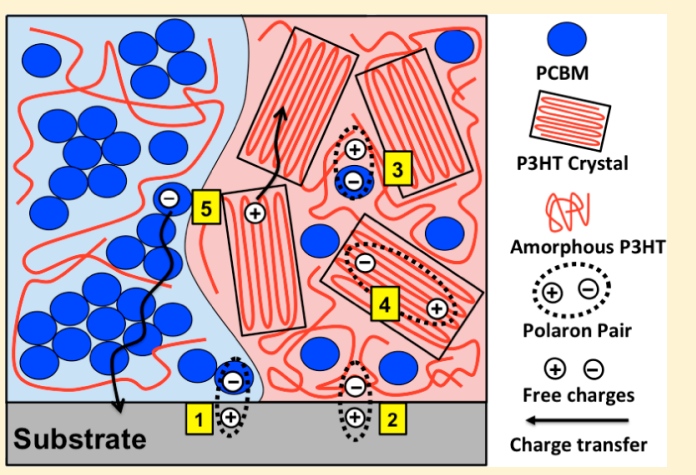
polymer-substrate interface (F1 and F2), the P3HT:PCBM charge transfer state (F3), the self-ionized (CT) state of P3HT (F4), and the band gap transition of P3HT (F5). This interpretation is also consistent with molecular orbital energy diagrams and electron microscopy-derived topological maps of the films. Photovoltage sign and substrate dependence can be understood with the depleted semiconductor model. Features F1-4 are caused by polarization of electrostatically bound charge pairs by the built-in electric field at the substrate- $\mathrm{BHJ}$ interface, whereas F5 is due to transport of free charge carriers through the film and through the substrate film interface. This work will promote the understanding of photochemical charge generation and transport in organic photovoltaic films.

\section{INTRODUCTION}

Since its initial discovery in 1992 by Hiramoto, ${ }^{1}$ the bulk heterojunction $(\mathrm{BHJ})$ organic photovoltaic (OPV) device has been continuously improved. ${ }^{2-7}$ The large interface in BHJs maximizes contact between the organic semiconductors and enhances charge separation of the relatively short-lived exciton pairs. $^{8-11}$ Charge separation is mediated by so-called transfer (CT) states that form as a result of the electronic interactions between P3HT and PCBM. The excitation energies of these states are below the band gap of the component materials, and they determine the maximum open circuit voltage $\left(V_{\text {oc }}\right)$ of the solar cell. ${ }^{12}$ In optical absorption spectra CT states are difficult to observe due to their low absorption cross section, but they have been detected using other more sensitive techniques. ${ }^{8,13-17}$ Additional midgap states at the polymer-electrode interfaces can result from chemical reactions with oxygen or from adsorption of solvent molecules. ${ }^{18-21}$ Such states have been observed with ultraviolet photoemission spectroscopy and inverse photoemission spectroscopy. ${ }^{22-25}$ They can affect photocurrent by trapping charge carriers or by inducing electric fields. ${ }^{26-30}$ We recently applied surface photovoltage spectroscopy (SPS) for the detection of CT and interface states in films of regioregular P3HT. ${ }^{31}$ SPS uses a semitransparent Kelvin probe that contactlessly probes the surface potential change of an illuminated film (Figure 1) versus the excitation energy. ${ }^{32,33}$ Measurable signals can be due to motion of free charge carriers through the film or the interface, due to polarization of charge
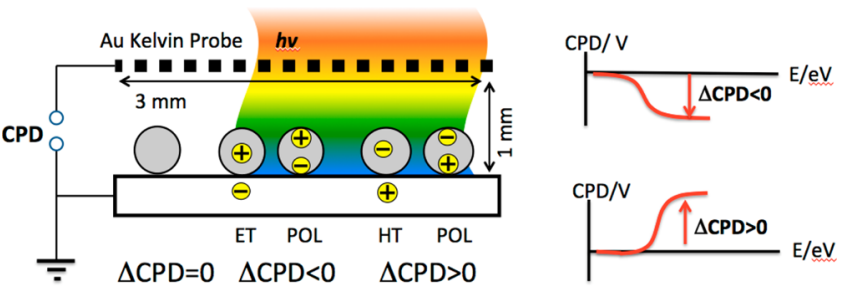

Figure 1. Geometry of SPV measurement and example spectra. Contact potential changes are generated by polarization (POL) or electron/hole transfer $(\mathrm{ET} / \mathrm{HT})$ into the substrate. A negative $\Delta \mathrm{CPD}$ signal corresponds to electron movement toward the substrate. The $\triangle \mathrm{CPD}$ value can be interpreted as the voltage that develops between the film and the substrate under illumination.

Received: January 8, 2014

Revised: June 5, 2014

Published: June 6, 2014 
carrier pairs (polarons) or due to the injection of charge carriers into molecular adsorbates. ${ }^{34}$ Since the technique is potentialsensitive and not based on photocurrents, even low densities of states and their excitation energies can be observed. ${ }^{34-39}$

Here we use the technique on films of $(6,6)$-phenyl- $\mathrm{C}_{61}$ butyric acid methyl ester (PCBM), ${ }^{10,40-43}$ and on bulk heterojunction films formed by self-assembly of PCBM and the polymer poly(3-hexylthiophene) (P3HT). SPV spectra were recorded as a function of substrate material, film annealing conditions, and film thickness, and using amorphous $\mathrm{TiO}_{x}$ and calcium niobate interlayers. ${ }^{44}$ Five photovoltage features were detected that can be assigned to interface states, to electronic transitions of $\mathrm{P} 3 \mathrm{HT}$, and to the $\mathrm{P} 3 \mathrm{HT}: \mathrm{PCBM}$ charge transfer state. Within the PCBM films, two low-energy optical transitions can be assigned to interfacial states and direct excitation of PCBM. The size and sign of the low-energy photovoltage features agree with the depleted semiconductor model, while the high-energy feature can be explained by diffusive charge transport.

\section{EXPERIMENTAL DETAILS}

Sample Preparation. Poly-3-hexylthiophene (P3HT) with M.W. $\approx 75 \mathrm{kDa}$ and a reported regioregularity of $\geq 95 \%$ was obtained from Plextronics. (6,6)-Phenyl- $\mathrm{C}_{61}$-butyric acid methyl ester (PCBM) was purchased from Nano-C. The aqueous suspension of PEDOT:PSS (Clevios P VP Al 4083) with resistivity between 500 and $5000 \Omega \mathrm{cm}$ was filtered with an $0.45 \mu \mathrm{m}$ Teflon filter before coating. Tetrabutylammoniumcapped calcium niobate nanosheets (CNO) were prepared according to published procedures. ${ }^{45,46}$ Before depositing, the nanosheets were centrifuged and redispersed three times in absolute ethanol and four times in deionized $18 \mathrm{M} \Omega$ water. A $\mathrm{TiO}_{x}$ sol-gel was synthesized from titanium isopropoxide (TIP, 97\%, Sigma-Aldrich) according to published procedure ${ }^{47}$ and diluted in methanol for spin-coating. Polymer films were prepared by first dissolving $\mathrm{P} 3 \mathrm{HT}$ and $\mathrm{PCBM}$ in chlorobenzene $(20 \mathrm{mg} / \mathrm{mL})$ and heating at $60{ }^{\circ} \mathrm{C}$ overnight. An $80 \pm 5 \mathrm{~nm}$ thick film of 1:1 P3HT:PCBM BHJ (PCBM-only, $60 \pm 5 \mathrm{~nm}$ thick) was prepared by spin-coating in an inert nitrogen atmosphere onto a 1.0 in. $^{2}$ commercial-grade indium-tin oxide (ITO) coated glass slide. Thicker BHJ films $(250 \pm 20 \mathrm{~nm})$ were prepared with the same procedure using twice the concentration $(40 \mathrm{mg} / \mathrm{mL})$ and spinning at $50 \%$ reduced speed. Nanofiber $\mathrm{BHJ}$ films were prepared by spin coating a toluene solution of 1:1 PCBM and PH3T J-aggregate nanofibers (the latter prepared separately using the known procedure $^{48}$ ) at a speed of $600 \mathrm{rpm}$. The nanofibers were several micrometers in length and $15 \mathrm{~nm}$ in diameter. The ITO (ca. $140 \mathrm{~nm}$ thick and $15 \Omega$ sheet resistance) was cleaned before use by ultrasonicating for $10 \mathrm{~min}$ in acetone, 2-propanol, and then water, respectively. Immediately before use the ITO was exposed to UV-ozone for $30 \mathrm{~min}$ and then transferred directly to inert atmosphere for deposition. Silver-coated glass substrates $(100 \mathrm{~nm})$ were prepared in-house through vapor deposition. When indicated, an interlayer of PEDOT:PSS (40 $\mathrm{nm})$ or CNO $(15 \mathrm{~nm})$ or $\mathrm{TiO}_{x}(20 \mathrm{~nm})$ were spin-coated onto the ITO substrate and heated at $110-150{ }^{\circ} \mathrm{C}$ for $5 \mathrm{~min}$ to remove any remaining solvent before depositing the $\mathrm{BHJ}$ layer. To test the effect of heat treatment, sample annealing was performed at $110{ }^{\circ} \mathrm{C}$ for $15 \mathrm{~min}$ under nitrogen atmosphere.

Measurements. UV-vis absorption spectra were recorded with a Thermo Scientific Evolution 220 UV-vis spectrophotometer equipped with an integrating sphere. Film thicknesses were measured with a Dektak surface profiler calibrated to a $\mathrm{Si}-\mathrm{SiO}_{2}$ ellipsometry standard. Monochromatic light intensity measurements were performed using an International Light Technologies IL400BL photometer with an IR-visible detector. SPV measurements were conducted inside a custom-built highvacuum measurement chamber connected to a Pfeiffer HiCube 80 Eco turbo pump and a Besocke Delta Phi GmbH Kelvin probe control unit. Contact potential difference (CPD) signals were measured using a vibrating Kelvin probe (Au grid, $3 \mathrm{~mm}$ diameter, $60 \%$ transparent) and read out with a Keithley 2700 voltmeter that was connected to a PC. Light was generated by $175 \mathrm{~W} \mathrm{Xe}$ arc lamp and filtered through a monochromator (Oriel Cornerstone 130). The light power density at the sample was $<1 \mathrm{~mW} \mathrm{~cm}{ }^{-2}$. Note that our previously reported photoflux values were incorrect. ${ }^{31}$ The $\mathrm{x}$-axis values in Figure $7 \mathrm{~B}$ in reference 31 should be by a factor of $10^{9}$ smaller. The correct $\mathrm{x}$ axis value range is $1 \times 10^{14}-1 \times 10^{17}$. Film samples were mounted inside the vacuum chamber, $1.0 \mathrm{~mm}$ underneath the Kelvin probe, the chamber was evacuated to $10^{-7} \mathrm{bar}$, and spectra were obtained by scanning from low to high energy in $100 \mathrm{~cm}^{-1}$ steps with a period interval of $5 \mathrm{~s}$. Light spectra were corrected for background effects (drift, desorption of gas and solvent molecules) by subtracting a prior background scan conducted in the dark. The spectra plot the contact potential difference (CPD) signal versus photon energy. The CPD value is the difference in work functions between the sample film and the gold Kelvin probe, plus contributions from surface and interface dipoles. $^{31,33}$ A positive CPD change corresponds to a photodipole with a negative pole at the surface and the positive side toward the back electrode. High-angle annular dark-field electron tomography (HAADF-ET) data were acquired with an JEOL 2100F electron microscope at $200 \mathrm{kV}$. Reconstructions were performed using custom code in MATLAB (MathWorks) utilizing the DART algorithm. The reconstruction minimized the difference between the forward-projected reconstruction and the original tilt series, yielding a material-labeled reconstruction that closely matched the original data. A twodimensional vertical slice of the original $3 \mathrm{D}$ data is presented in Figure 5 (viewing a small cross section of a $\mathrm{BHJ}$ ).

\section{RESULTS AND DISCUSSION}

Figure 2A shows optical absorption and SPV spectra of a pristine PCBM film deposited onto ITO.

The optical spectrum is similar to other literature reports for PCBM $^{49}$ and $\mathrm{C}_{60}$ films. ${ }^{50}$ The absorption edge at $1.75 \mathrm{eV}$ has been previously assigned to charge transfer (CT) states formed by the interaction between fullerenes within the film. ${ }^{29,49,51-54}$ With thick films, a weak absorption tail out to $1.55 \mathrm{eV}$ can be seen. ${ }^{49}$ The small sharp $1.75 \mathrm{eV}$ absorption is due to the formation of a "Frenkel exciton" (electron hole pair within the same fullerene). ${ }^{29,49}$ The direct excitation of this state is forbidden by the selection rules of the icosahedral fullerene, ${ }^{55,56}$ but emission from this state can be observed in photoluminescence spectra of PCBM. ${ }^{49}$ The SPV spectrum of the PCBM film contains a negative feature at $1.1-1.4 \mathrm{eV}$ and a positive one at $1.4-2.3 \mathrm{eV}$. The fine structure of feature 1 (F1) is caused by the specific emission lines of the Xe arc lamp, as detailed in our earlier paper. ${ }^{31}$ Because of its low intensity and low excitation energy, F1 is assigned to interface charge transfer (ICT) states at the PCBM-substrate interface. As the diagram in Figure $2 \mathrm{~B}$ shows, the PCBM HOMO and the ITO conduction band edges have a $1.3 \mathrm{eV}$ band offset in the region of the photovoltage signal. Excitation of these states would 

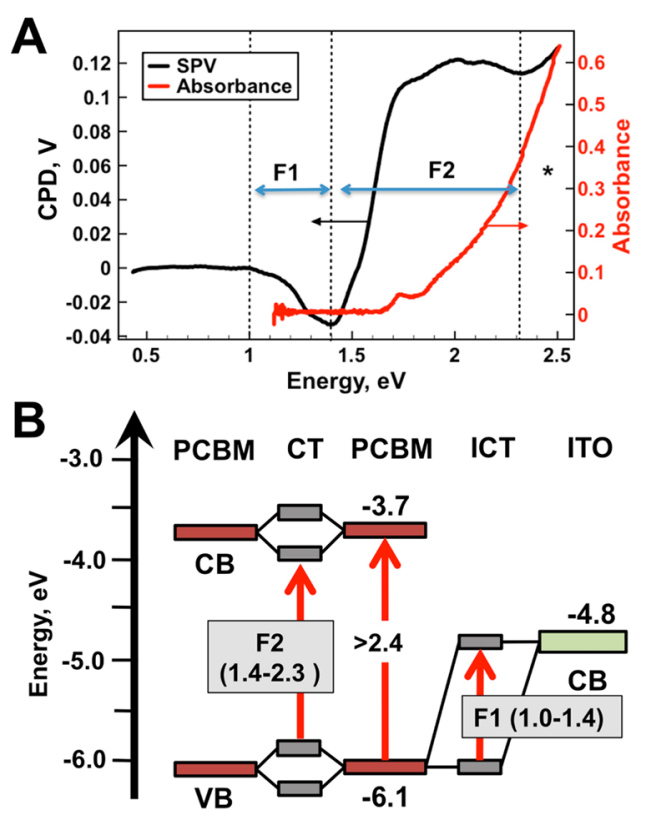

Figure 2. (A) SPV (black) and UV-vis absorption (red) spectra of a $60 \mathrm{~nm}$ PCBM film thick on ITO. *Instrument artifact. (B) Proposed energy diagram for PCBM with electronic transitions.

cause charge transfer to the ITO conduction band edge, which agrees well with the observed negative $\triangle \mathrm{CPD}$ signal. Transitions at $1.0 \mathrm{eV}$ have been previously observed in SPV spectra of $\mathrm{C}_{60}$ films, but the nature of these midgap states was not determined. ${ }^{57}$ For P3HT:PCBM BHJ devices, midgap states have been associated with a lowering of the charge transfer rate and reduced electrical output. ${ }^{58}$ The $1.4-2.3 \mathrm{eV}$ energy range for F2 in the SPV spectrum agrees with both the Frenkel exciton in PCBM and with the beginning of the PCBM-PCBM CT transition at $1.75 \mathrm{eV}$. As the formed charge carries are not free, the observed photovoltage must be due to polarization of these electron hole pairs by the electric field at the substrate-PCBM interface (see depleted semiconductor model in Figure 8). Excitation $>1.8 \mathrm{eV}$ increases the exciton concentration in the film (see absorption spectrum) but does not raise the photovoltage signal further. This suggests that the electric field at the substrate-PCBM interface has already been neutralized by electron-hole pairs created by excitation at $<1.8$ $\mathrm{eV}$.

Next we turn to the observation of excited states in thermally annealed $\mathrm{BHJ}$ films containing $(1: 1 \mathrm{w} \%)$ regioregular P3HT and PCBM. Figure $3 \mathrm{~A}$ shows absorbance, fluorescence, and SPV spectra for such a film. Both the optical absorbance at 2.0 $\mathrm{eV}$ and the fluorescence at $1.7 \mathrm{eV}$ belong to the excitation of the P3HT band gap and P3HT charge transfer (or autoionized) states, respectively. These features are also observed in films of pure P3HT (see earlier paper). ${ }^{31}$ They obscure the weaker optical features of PCBM (Figure 2A). No additional bands from a possible $\mathrm{PCBM}-\mathrm{P} 3 \mathrm{HT}$ interaction are observed in the optical spectra. On the other hand, the SPV spectrum for the $\mathrm{BHJ}$ film differs substantially from the spectra of the isolated materials (red and blue traces in Figure $3 \mathrm{~B}$ ). ${ }^{31}$ The observed features are labeled F1 through F5, in Figure 3C, according to photon energy. Feature 1 is a weak signal between 0.75 and 0.9 $\mathrm{eV}$, whose sign and magnitude depends on the substrate. Feature 2 appears between 0.9 and $1.3 \mathrm{eV}$ and is the strongest signal in the spectrum ( $85 \mathrm{mV}$ at its peak). Depending on the
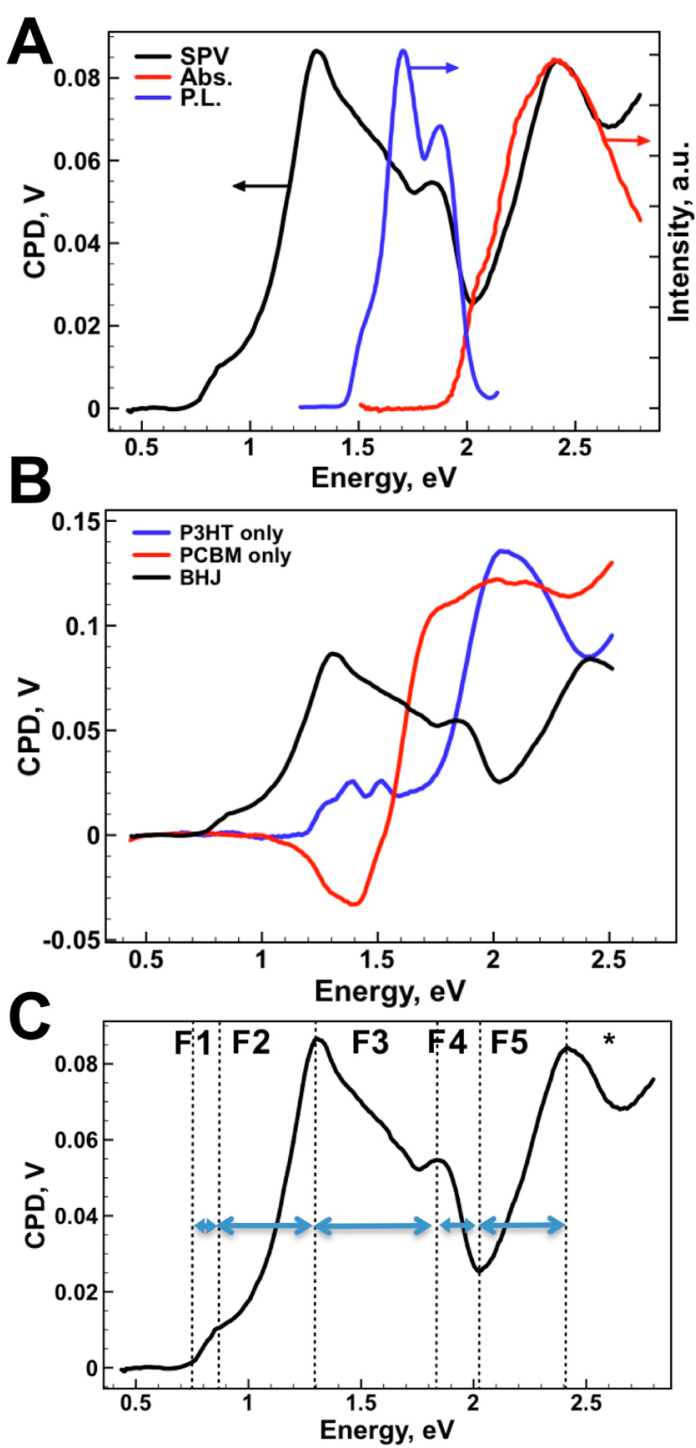

Figure 3. (A) SPV, UV-vis absorption, and photoluminescence (PL) spectra of an $80 \mathrm{~nm}$ thermally annealed P3HT:PCBM BHJ film on ITO. (B) Comparison of SPV spectra for films of P3HT $(120 \mathrm{~nm})$, PCBM $(60 \mathrm{~nm})$, and 1:1 wt \%P3HT:PCBM BHJ (ca. $80 \mathrm{~nm}$ thick). All films were deposited on ITO and thermally annealed. (C) BHJ SPV spectrum with numbered features. *Instrument artifact.

substrate, this signal can be positive (ITO, PEDOT:PSS) or negative $\left(\mathrm{Ag}, \mathrm{TiO}_{x}, \mathrm{CNO}\right)$. The signal turns into feature 3 at $1.3-1.8 \mathrm{eV}$. F3 also depends on the substrate work function, as will be shown below. It ends with a very small positive CPD change at $1.8 \mathrm{eV}$ that is observed only on the ITO substratefor that reason this small feature is not assigned a separate number. It may be associated with the Frenkel exciton observed in the optical spectrum for PCBM, as described above. Feature 4 lies between 1.80 and $2.0 \mathrm{eV}$. The sign of feature 4 is also substrate work function-dependent. The feature disappears in $\mathrm{BHJ}$ films with regiorandom instead of regioregular P3HT (Figure $\mathrm{S} 1$ in the Supporting Information). Feature 5 (blue) occurs between 2.0 and $2.4 \mathrm{eV}$ and is observed with positive (Ag, ITO) or negative (PEDOT:PSS, CNO, $\mathrm{TiO}_{x}$ ) sign, depending on the substrate. Lastly, the positive SPV signal at $\geq 2.4 \mathrm{eV}$ does not originate from the polymer film but is an artifact of the instrument. ${ }^{31}$ It is also observed in background 


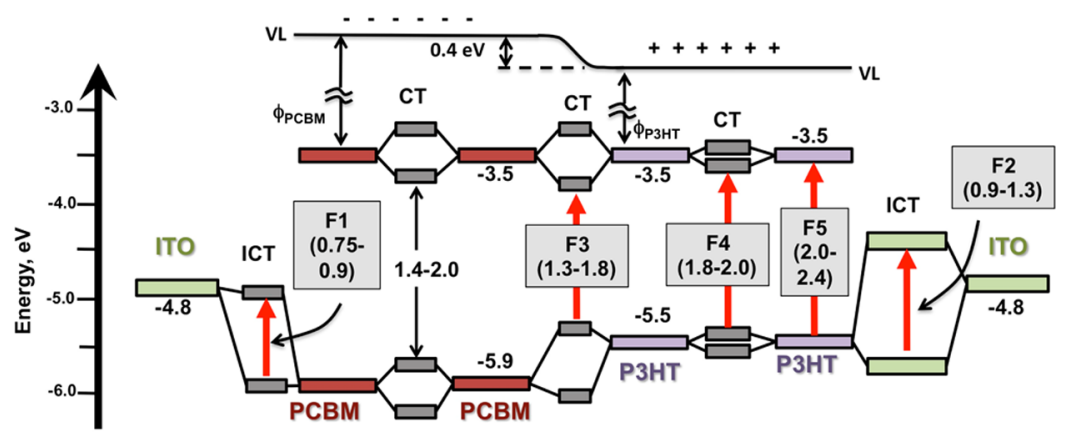

Figure 4. Energy diagram for P3HT:PCBM films on ITO. Shifts in the local vacuum levels are due to autoionization of P3HT donor and PCBM acceptor. $^{60}$

spectra on non-coated substrates and will not be discussed further.

Based on their excitation energy, F1-F5 can be assigned to specific transitions in the bulk heterojunction film and at the interfaces, as shown in the orbital energy diagram in Figure 4. Transitions 4 and 5 have been previously observed in pristine P3HT films and assigned to excitation of the autoionized (CT state, at $1.7 \mathrm{eV}$ ) and to the band gap excitation of P3HT (2.0 $\mathrm{eV}){ }^{31}$ This assignment agrees well with the fluorescence emission that is known to involve the P3HT-CT (autoionized) state and the optical absorption from band gap excitation of $\mathrm{P}_{3 \mathrm{HT}}{ }^{31}$ It also agrees with the finding that F4 disappears in the SPV spectrum for a $\mathrm{BHJ}$ film made with regiorandom (rra) instead of regioregular P3HT (Figure S1 in the Supporting Information). The rra isomer of P3HT has weaker chain interactions and lower crystallinity than $\mathrm{rr}-\mathrm{P} 3 \mathrm{HT}$; ${ }^{59}$ correspondingly, the rra-P3HT-CT state concentration is reduced, and the fluorescence is quenched.

Feature 3 does not occur in separate films of PCBM or P3HT. The observed $1.3-1.8 \mathrm{eV}$ energy range for this transition is close to the $\operatorname{HOMO}(\mathrm{D})-\mathrm{LUMO}(\mathrm{A})$ separation of separate donor and acceptor $(5.3-3.7=1.6 \mathrm{eV})$. This suggests that feature 3 is due to excitation of the P3HT-PCBM charge transfer (CT) state in the $\mathrm{BHJ}$ film, as shown in the energy diagram. Because the transition probability of the $\mathrm{D}-\mathrm{A}$ CT state excitation is low, it is not observed in the optical excitation spectrum of the film. The energy gap of the D-A CT transition controls the $V_{\text {oc }}$ of the device ${ }^{10,12,14}$ and thus is important for the function of BHJ solar cells. Further evidence for this assignment comes from the SPV spectrum for a $\mathrm{BHJ}$ film made of P3HT nanofibers and PCBM (Figure S2 in the Supporting Information). Due to the nanofiber morphology of $\mathrm{P} \mathrm{HT},{ }^{48}$ that film is depleted of P3HT-PCBM contacts, and thus F3 is not observed.

As can be seen from the energy diagram in Figure 4, the interaction between $\mathrm{P} 3 \mathrm{HT}$ and PCBM results in the splitting of HOMO and LUMO orbitals of the donor and the acceptor, which explains the 1.3-1.8 eV energy range of the transition. In addition, dark state charge transfer (autoionization) between donor and acceptor occurs as a result of the electrochemical equilibrium between these molecules. This shifts the PCBM frontier orbitals $0.2 \mathrm{eV}$ to reducing potentials (negative charging) and the $\mathrm{P} 3 \mathrm{HT}$ orbitals $0.2 \mathrm{eV}$ to oxidizing potentials (due to positive charging) and modifies the local vacuum levels as shown in Figure 4. Shifts of the frontier orbitals in P3HT/ PCBM blends have been previously observed with photoelectron spectroscopy and Kelvin probe microscopy. ${ }^{60,61}$
As mentioned above, we attribute feature 2 at $0.9-1.3 \mathrm{eV}$ to excitation of interfacial states at the $\mathrm{P} 3 \mathrm{HT}$-substrate interface. In our earlier SPV measurements on pure P3HT films, these states occur at higher energy $(1.2-1.7 \mathrm{eV}) .{ }^{31}$ The shift in energy in the mixed phase is attributed to the electrochemical equilibrium with PCBM, as mentioned above, which moves the P3HT states to more oxidizing potentials and changes the overlap with the substrate orbitals at the interface. For PCBM, the energy levels shift in the opposite direction, reducing the separation between PCBM HOMO and ITO conduction band. This shifts the PCBM-ITO interfacial states (F1) to 0.75-0.9 $\mathrm{eV}$. As should be expected for interface-specific assignments, F1 and F2 strongly depend on the substrate work function and the sample processing conditions (see below).

Figure 5 maps the observed SPV features to distinct spatial regions in the polymer film. According to electron tomography

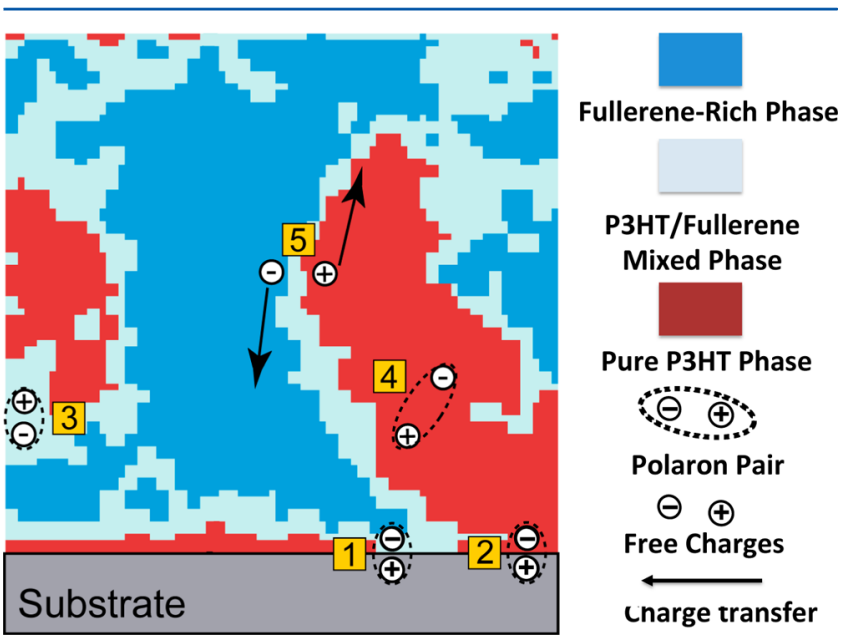

Figure 5. Concentration-specific $3 \mathrm{D}$ morphology of a $\mathrm{BHJ}$ film measured using HAADF-ET. ${ }^{62}$ Assignment of photovoltage features F1-5 is shown with respect to the location within the films. HAADFET does obtain contrast from density changes only, so no assignment of crystallinity is made here.

data, ${ }^{62,63}$ thermally annealed $\mathrm{BHJ}$ films contain three different phases, a fullerene-rich amorphous phase ( $10 \%$ P3HT by volume), a $\mathrm{P} 3 \mathrm{HT}-$ fullerene mixed amorphous phase, and pure P3HT domains. The substrate surface has a higher density of the fullerene-rich and mixed phases, while the pure-P3HT domains are near the center of the film. ${ }^{62-64}$ This explains the presence of two types of interfacial SPV features (F1 and F2) corresponding to local interfacial states between the substrate and fullerene and amorphous $\mathrm{P} 3 \mathrm{HT}$, respectively. Feature 3 
occurs in the fullerene-P3HT mixed phase, which is dominated by CT interactions between P3HT and PCBM. ${ }^{63}$ This phase is reduced in the nanofiber P3HT/PCBM blend (Figure S2 in the Supporting Information), and correspondingly, F3 is not observed there. Feature 4 is expected to be most prominent in the crystalline regions of pure-P3HT domains where the concentration of P3HT CT states is highest. Finally, F5 (2.0-2.4 eV) involves band gap excitation of P3HT followed by the formation of free and mobile charges. The signal is associated with excitation of ordered P3HT domains and then of amorphous $\mathrm{P} 3 \mathrm{HT}$ or PCBM at higher excitation energy.

Having assigned all photovoltage features to energy states and locations in the film, we next examine the substrate dependence of the SPV spectra. As can be seen from Figure 6,
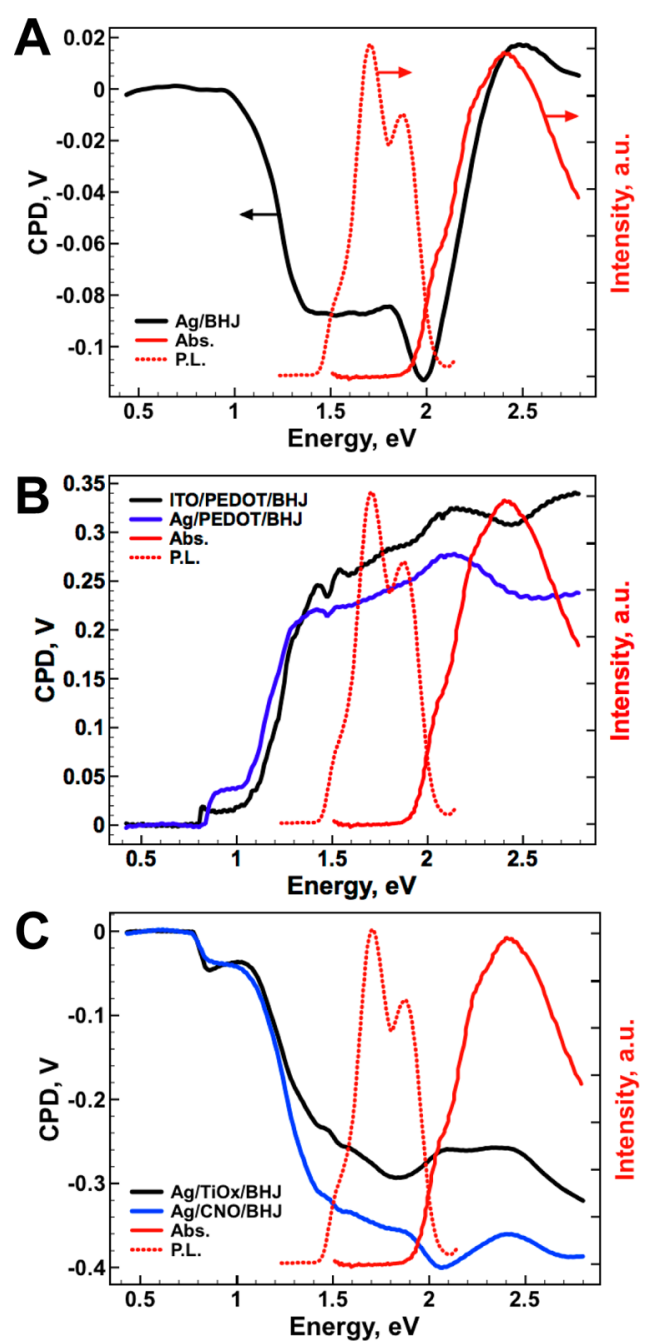

Figure 6. SPV spectra of P3HT:PCBM BHJ films (ca. $80 \mathrm{~nm}$ thick, annealed at $110{ }^{\circ} \mathrm{C}$ for $15 \mathrm{~min}$ ) on various substrates: (A) silver; (B) PEDOT:PSS deposited on ITO; (C) colloidal $\mathrm{TiO}_{x}$ and CNO deposited on Ag.

size and direction of all features in the spectrum are influenced by the substrate. The most significant changes are the inversion of $\mathrm{F} 2$ in going from the ITO substrate (Figure 3A) to CNOcoated silver (Figure 6C) and the inversion of F5 in going from silver (Figure 6A) to PEDOT:PSS coated ITO (Figure 6B). Also, F1 and F3 disappear on the silver substrate (Figure 6A). In order to understand these spectral variations and their dependence on the substrate, all photovoltage signals were plotted in Figure 7 against the substrate work function.

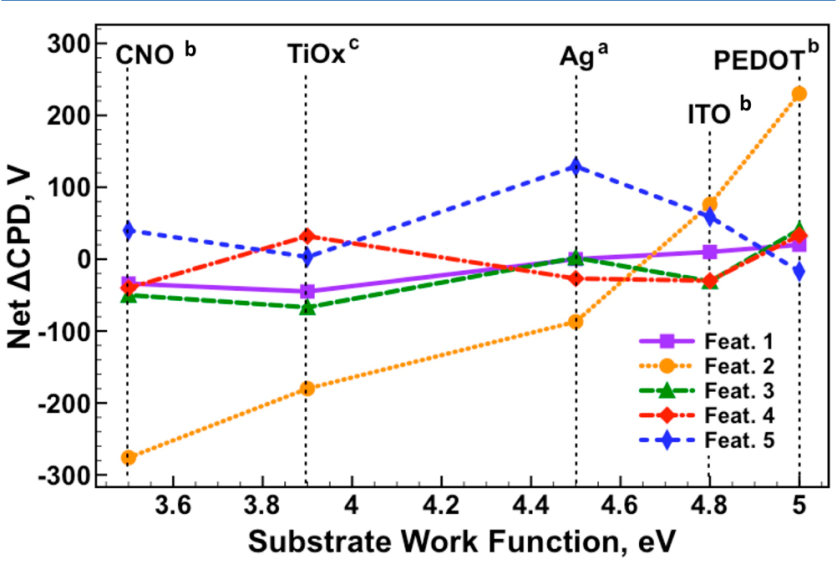

Figure 7. Photovoltage variation with substrate work function for a P3HT:PCBM BHJ film: (a) from Lide et al; ${ }^{75}$ (b) from Chang et al; ${ }^{44}$ (c) from $\mathrm{Xu}$ et $\mathrm{al}^{76} \mathrm{~A}$ linear relationship between $\triangle \mathrm{CPD}$ and the substrate work function would be expected if charge transfer was driven by the built-in potential of the substrate-polymer junction. F1 and F2 approach this situation.

Features 1 and 2, and to some extent, F3, show a monotonic increase with the work function of the substrate. In contrast, features 4 and 5 exhibit no clear trend. These observations can be rationalized with the depleted semiconductor model in Figure 8. According to this model, charge carriers in the excited $\mathrm{BHJ}$ film move under a built-in electric field that results from the electrochemical equilibrium at the substrate-polymer interface and from (unknown) dipoles at the interface. ${ }^{31,33,65-68}$ The built-in field derives from the potential drop $V_{\mathrm{bi}}$ within the space charge layer of the film. The potential drop originates from the initial difference of Fermi levels in substrate $E_{\mathrm{F}}(\mathrm{Sub})$ and polymer film $E_{\mathrm{F}}(\mathrm{BHJ})$ before electrical contact is made: $e V_{\mathrm{bi}}=E_{\mathrm{F}}(\mathrm{Sub})-E_{\mathrm{F}}(\mathrm{BHJ})$. Based on the inversion of the polarity of the $\mathrm{F} 2$ signal in Figure 7 , the Fermi level $E_{\mathrm{F}}$ of the polymer can be estimated as $-4.7 \mathrm{eV}$, slightly above the $E_{\mathrm{F}}$ of ITO $(-4.8 \mathrm{eV})$. The depleted semiconductor model is supported by impedance spectroscopy, ${ }^{69,70}$ capacitance measurements, ${ }^{28,71}$ and time-of-flight electron-collection. ${ }^{72}$ Furthermore, XPS and UPS studies also confirm an electrochemical equilibrium between P3HT:PCBM and substrate. ${ }^{25,26,30,73,74}$ In these earlier studies, the polymer film was observed to be mildly p-type, as a result of exposure to oxygen and water during film preparation. On the contrary, the films described here are clearly mildly n-type, on the basis of the SPV data and the model in Figure 8. This is due to the exclusion of oxygen during the SPV measurements and during film preparation (see Experimental section).

According to Figure 8 , the surface photovoltage in the P3HT:PCBM film is generated by polarization of electrostatically coupled electron-hole pairs under the built-in field (F14) or by transfer of free charge carriers through the film and across the polymer-substrate interface (F5). Because F1 and F2 are excited first during the monochromatic scan, they are most strongly controlled by the built-in field. However, as increasing concentrations of charge carriers build up and polarize the film, the interfacial electric field is attenuated, and the driving force for charge separation at higher energy excitation is diminished. As a result, F3 and F4 are less 


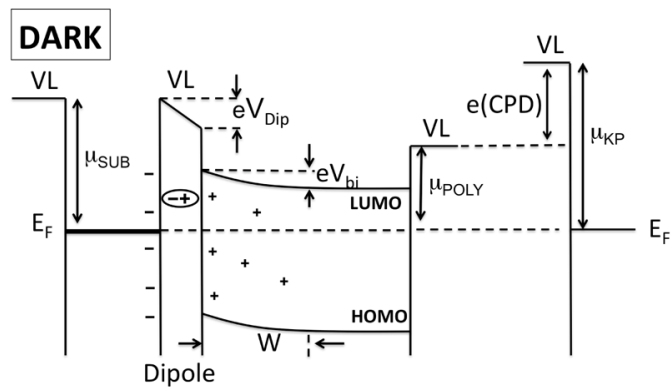

Substrate

Polymer

Kelvin Probe

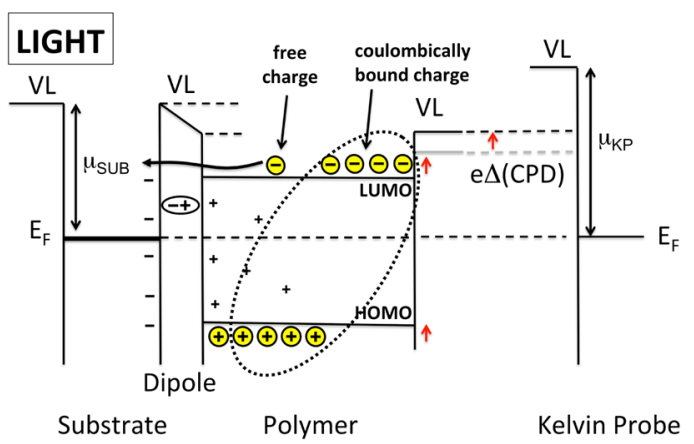

Figure 8. Depleted semiconductor model, after Ishii, ${ }^{66}$ showing effect of Fermi level equilibration and interfacial dipoles on the potential distribution through the polymer film in the dark. With ITO or PEDOT:PSS substrates the bands bend down into the film, as shown, but with $\mathrm{CNO}, \mathrm{TiO}_{x}$, and $\mathrm{Ag}$ as substrates, the bands bend up (not shown). Under illumination, photovoltages $\triangle \mathrm{CPD}$ are generated via polarization of coulombically bound charge carriers (F1-4, inside dotted line) and via transfer of free charge carriers (F5). These lightinduced processes alter the local vacuum level (VL) at the P3HT surface. $\mathrm{w}$ : space charge layer width, $\mu_{\mathrm{i}}$ : work functions of polymer, substrate, and Kelvin probe, $V_{\mathrm{bi}}$ : built in voltage, $V_{\mathrm{Dip}}$ : potential drop from interfacial dipole, $E_{\mathrm{F}}$ : Fermi level.

correlated with $E_{\mathrm{F}}(\mathrm{Sub})$. Their polarization becomes dependent on local dipoles that arise from polymer crystal orientation in the film and from autoionization at the P3HT-PCBM interface (see also Figure 5).

Feature 5, finally, evolves from free charge carriers that can diffuse across the film and pass through the polymer-substrate interface. Their movement is controlled by the residual field, by the local concentrations of free charge carriers, by the morphology of the film, and by the kinetics of interfacial charge transfer. ${ }^{77}$ Therefore, no simple correlation of F5 with the substrate work function is observed. The model in Figures 4,5 , and 8 can also be used to explain the effects of thermal treatment and film thickness on the photovoltage as shown in Figure 9. Before annealing, the spectrum contains at least eight weak features (bends) and only one strong feature at 1.2-1.7 $\mathrm{eV}$ (Figure 9A). After annealing $\left(110^{\circ} \mathrm{C}\right.$ for $\left.15 \mathrm{~min}\right)$, the number of features is reduced to the characteristic five ones discussed above. It is well-known that annealing improves the order and crystallinity of the $\mathrm{BHJ}$ system ${ }^{78-80}$ and increases the fullerene-rich and the crystalline P3HT phases at the expense of the P3HT-PCBM mixed phase. Consequentially, features 4 and 5 belonging to the $\mathrm{CT}$ and band gap excitation of $\mathrm{P} 3 \mathrm{HT}$ become more dominant, due to crystallization of P3HT and improved charge transport. Feature 3 also becomes more recognizable, in agreement with a homogenization of the P3HT-PCBM mixed phase. Feature 1 disappears upon annealing, which could be a result of a vertical concentration
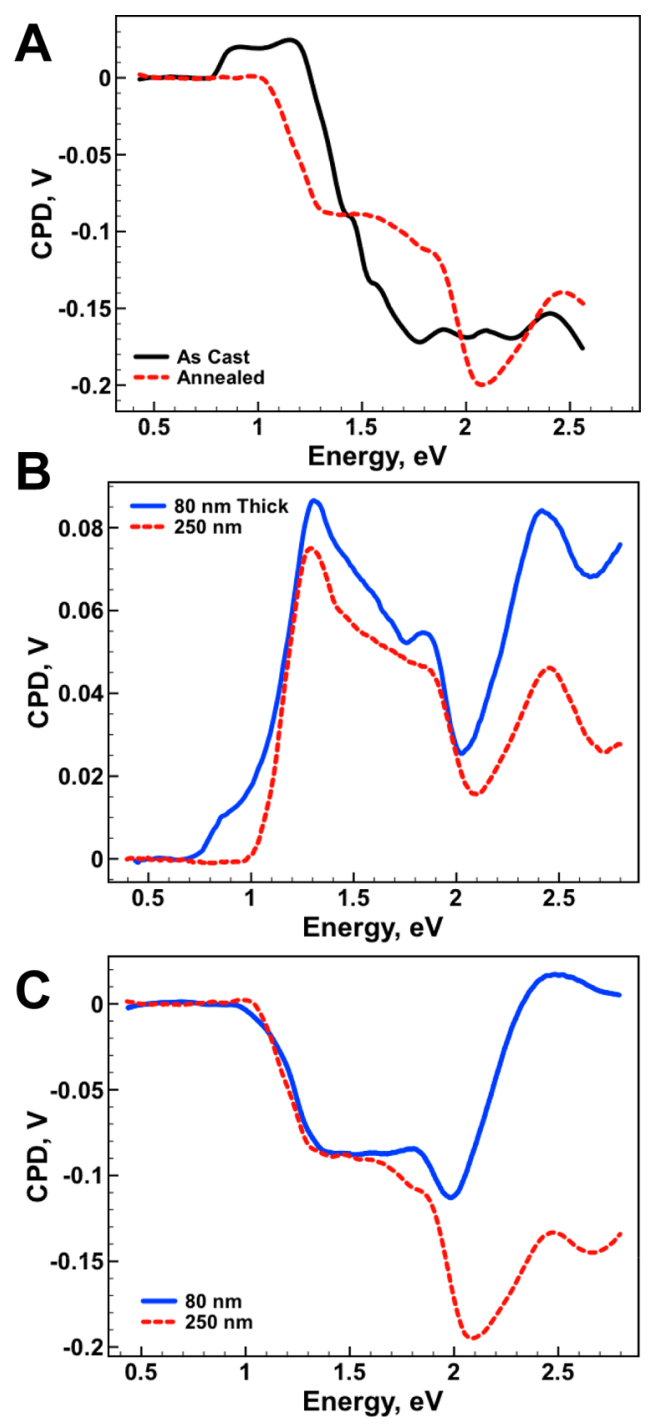

Figure 9. (A) SPV spectra of a ca. $250 \mathrm{~nm}$ thick P3HT:PCBM BHJ film on $\mathrm{Ag}$ as cast and after annealing at $110^{\circ} \mathrm{C}$ for $15 \mathrm{~min}$. (B) SPV spectra of thermally annealed P3HT:PCBM BHJ films of variable thickness on ITO and (C) on Ag.

change of the substrate-polymer interface. ${ }^{64}$ Neutron reflectivity measurements support vertical segregation of PCBM and P3HT phases on Ag substrates upon heating. ${ }^{64}$ Also, F2 is reduced by annealing, further suggesting that it originates from the $\mathrm{P} 3 \mathrm{HT}$ mixed phase-substrate interface.

The influence of film thickness was examined with 80 and $250 \mathrm{~nm}$ thick $\mathrm{BHJ}$ films on silver and ITO substrates (Figure 9A-C). On ITO (Figure 9B) increasing the thickness causes F1 to disappear and F5 to become weaker, while features 2, 3, and 4 remain unchanged. Presently, there is no clear explanation for the disappearance of F1. It might be linked to a change of the morphology of the thicker $\mathrm{BHJ}$ film that reduces the amount of PCBM at the substrate. However, in general the PCBM concentration at the bottom electrode has been found to be less dependent on film thickness than annealing conditions and solvents. ${ }^{64}$ The fact that features 2,3 , and 4 do not increase with film thickness confirms that these signals are controlled by the built-in electric field. In contrast, the reduction of F5 in the thicker film clearly suggests a dependence on the ability of the holes to travel to the back contact and to inject into the substrate. This is more difficult in 
thicker films, where recombination competes more effectively with charge transport. Also, because of light attenuation above $2.0 \mathrm{eV}$, charge carriers are created farther away from the back interface. In active OPV devices, direct band gap excitation of the donor (F5) is responsible for generating photovoltage.

Figure 9C shows the effect of film thickness for a silver substrate. On silver, F1 is no longer observed, as mentioned above, and F2 has the opposite polarity than in ITO. This is due to the lower work function of Ag, which reverses the polarity of the built-in voltage (Figure 7). Increasing the $\mathrm{BHJ}$ film thickness does not affect F2 and only mildly affects F3 because both are controlled by the (constant) built-in field. With a constant field, the increase of F4 for the thicker film must be attributed to a change of the film morphology or a greater volume fraction of P3HT crystals. Such a change would promote the P3HT CT (autoionized) state in these crystals or at their interfaces. Similar to the ITO substrate, F5 decreases in the thicker film, supporting the conclusion that F5 is limited by diffusive charge transport to the back electrode.

\section{CONCLUSION}

In summary, we present the first surface photovoltage spectroscopy study on pristine PCBM and P3HT:PCBM $\mathrm{BHJ}$ films. In PCBM films, two photovoltage features are observed at $1.0-1.4 \mathrm{eV}$ and at $1.4-2.3 \mathrm{eV}$, which can be assigned to generation of electrostatically coupled polaron pairs at the interface and in the bulk. In annealed $\mathrm{BHJ}$ films five distinct photovoltage features are observed. These can be associated with interface states (F1 and F2), the P3HT:PCBM transfer state (F3), the self-ionized (CT) state of P3HT (F4), and the band gap transition P3HT (F5), respectively. Sub-band gap excitations (F1-F4) lead to electrostatically coupled polaron pairs that are polarized by the built-in electric field at the substrate-BHJ interface, thus generating the SPV signal. Charge carriers formed under band gap excitation (F5) produce a photovoltage via charge transfer in the film and across the polymer-substrate interface. The photovoltage sign, size, and dependence of F1-5 on substrates, film thickness, and thermal annealing state of the films can be explained with the depleted semiconductor model. The ability of SPS to sensitively observe the formation and interplay of polarons and free charge carriers in films of organic semiconductors is significant for the understanding of charge transport in such films. It will benefit the development of organic photovoltaics and light emitting devices.

\section{ASSOCIATED CONTENT}

\section{S Supporting Information}

SPV, UV-vis absorption, and photoluminescence spectra of P3HT:PCBM BHJ films made from regiorandom and regioregular nanofiber P3HT. This material is available free of charge via the Internet at http://pubs.acs.org.

\section{AUTHOR INFORMATION}

\section{Corresponding Author}

*Phone: 1-530-754-6242. E-mail: fosterloh@ucdavis.edu.

\section{Notes}

The authors declare no competing financial interest.

\section{ACKNOWLEDGMENTS}

This work was supported by a grant of the National Science Foundation (NSF, CBET 933435) and by a Scialog grant from
Research Corporation for Science Advancement. J.D.R. acknowledges the U.C. Davis College of Engineering/NSF RESOURCE program for funding. We thank Plextronics Inc. for supply of high-quality P3HT.

\section{REFERENCES}

(1) Hiramoto, M.; Fujiwara, H.; Yokoyama, M. P-I-N Like Behavior in 3-Layered Organic Solar-Cells Having a Co-Deposited Interlayer of Pigments. J. Appl. Phys. 1992, 72, 3781-3787.

(2) Y You, J.; Dou, L.; Yoshimura, K.; Kato, T.; Ohya, K.; Moriarty, T.; Emery, K.; Chen, C. C.; Gao, J.; Li, G.; et al. A Polymer Tandem Solar Cell with $10.6 \%$ Power Conversion Efficiency. Nat. Commun. 2013, 4, 1446.

(3) Hadipour, A.; de Boer, B.; Blom, P. W. M. Organic Tandem and Multi-Junction Solar Cells. Adv. Funct. Mater. 2008, 18, 169-181.

(4) Deibel, C.; Dyakonov, V. Polymer-Fullerene BHJ Solar Cells (Review). Rep. Prog. Phys. 2010, 73, 096401.

(5) Clarke, T. M.; Durrant, J. R. Charge Photogeneration in Organic Solar Cells. Chem. Rev. 2010, 110, 6736-6767.

(6) Schlenker, C. W.; Thompson, M. E. The Molecular Nature of Photovoltage Losses in Organic Solar Cells. Chem. Commun. 2011, 47, 3702-3716.

(7) Mayer, A. C.; Scully, S. R.; Hardin, B. E.; Rowell, M. W.; McGehee, M. D. Polymer-Based Solar Cells. Mater. Today 2007, 10, $28-33$.

(8) Street, R. A.; Davies, D.; Khlyabich, P. P.; Burkhart, B.; Thompson, B. C. Origin of the Tunable Open-Circuit Voltage in Ternary Blend Bulk Heterojunction Organic Solar Cells. J. Am. Chem. Soc. 2013, 135, 986-989.

(9) Vandewal, K.; Gadisa, A.; Oosterbaan, W. D.; Bertho, S.; Banishoeib, F.; Van Severen, I.; Lutsen, L.; Cleij, T. J.; Van der Zande, D.; Manca, J. V. The Relation between Open-Circuit Voltage and the Onset of Photocurrent Generation by Charge-Transfer Absorption in Polymer: Fullerene Bulk Heterojunction Solar Cells. Adv. Funct. Mater. 2008, 18, 2064-2070.

(10) Vandewal, K.; Gadisa, A.; Tvingstedt, K.; Inganas, O.; Manca, J. V. On the Origin of the Open-Circuit Voltage of Polymer-Fullerene Solar Cells. Nat. Mater. 2009, 8, 904-909.

(11) Cowan, S. R.; Roy, A.; Heeger, A. J. Recombination in Polymer-Fullerene Bulk Heterojunction Solar Cells. Phys. Rev. B 2010, 82, 245207.

(12) Janssen, R. A. J.; Veldman, D. The Energy of Charge-Transfer States in Electron Donor-Acceptor Blends: Insight into the Energy Losses in Organic Solar Cells. Adv. Funct. Mater. 2009, 19, 19391948.

(13) Nelson, J.; Durrant, J. R.; Poplavskyy, D. CT in PCBM MDMOPPV. Adv. Funct. Mater. 2005, 15, 1171-1182.

(14) Tvingstedt, K.; Inganas, O.; Manca, J. V. Electroluminescence from Charge Transfer States in Polymer Solar Cells. J. Am. Chem. Soc. 2009, 131, 11819-11824.

(15) Dimitrov, S. D.; Bakulin, A. A.; Nielsen, C. B.; Schroeder, B. C.; Du, J. P.; Bronstein, H.; McCulloch, I.; Friend, R. H.; Durrant, J. R. On the Energetic Dependence of Charge Separation in Low-Band-Gap Polymer/Fullerene Blends. J. Am. Chem. Soc. 2012, 134, 1818918192.

(16) Behrends, J. Direct Detection of Photoinduced Charge Transfer Complexes in Polymer Fullerene Blends. Phys. Rev. B 2012, 85, 125206.

(17) Benson-Smith, J. J.; Goris, L.; Vandewal, K.; Haenen, K.; Manca, J. V.; Vanderzande, D.; Bradley, D. D. C.; Nelson, J. Formation of a Ground-State Charge-Transfer Complex in Polyfluorene/ 6,6-PhenylC-61 Butyric Acid Methyl Ester (PCBM) Blend Films and Its Role in the Function of Polymer/PCBM Solar Cells. Adv. Funct. Mater. 2007, 17, 451-457.

(18) Liang, Z. Q.; Gregg, B. A. Compensating Poly(3-hexylthiophene) Reveals Its Doping Density and Its Strong Exciton Quenching by Free Carriers. Adv. Mater. 2012, 24, 3258-3262. 
(19) Cook, S.; Furube, A.; Katoh, R. Matter of Minutes Degradation of Poly(3-hexylthiophene) under Illumination in Air. J. Mater. Chem. 2012, 22, 4282-4289.

(20) Manceau, M.; Rivaton, A.; Gardette, J. L.; Guillerez, S.; Lemaitre, N. The Mechanism of Photo- and Thermooxidation of Poly(3-hexylthiophene) (P3HT) Reconsidered. Polym. Degrad. Stab. 2009, 94, 898-907.

(21) Gregg, B. A. Charged Defects in Soft Semiconductors and Their Influence on Organic Photovoltaics. Soft Matter 2009, 5, 2985-2989.

(22) Kahn, A.; Koch, N.; Gao, W. Electronic Structure and Electrical Properties of Interfaces between Metals and $\pi$-Conjugated Molecular Films. J. Polym. Sci., Polym. Phys. Ed. 2003, 41, 2529-2548.

(23) Meyer, J.; Hamwi, S.; Riedl, T.; Kahn, A. Transition Metal Oxides for Organic Electronics: Energetics, Device Physics and Applications. Adv. Mater. 2012, 24, 5408-5427.

(24) Olthof, S.; Mehraeen, S.; Mohapatra, S. G.; Barlow, S.; Coropceanu, V.; Bredas, J. L.; Marder, S. R.; Kahn, A. Ultralow Doping in Organic Semiconductors: Evidence of Trap Filling. Phys. Rev. Lett. 2012, 109, 176601.

(25) Frisch, J.; Koch, N.; Neher, D. Full Electronic Structure across a Polymer Heterojunction Solar Cell. J. Mater. Chem. 2012, 22, 44181124.

(26) Hwang, J.; Wan, A.; Kahn, A. Energetics of Metal-Organic Interfaces: New Experiments and Assessment of the Field. Mater. Sci. Eng. 2009, 64, 1-31.

(27) Greiner, M. T. Universal Energy-Level Alignment of Molecules on Metal Oxides. Nat. Mater. 2012, 11, 76-81.

(28) Bisquert, J.; Garcia-Belmonte, G.; Munar, A.; Sessolo, M.; Soriano, A.; Bolink, H. J. Band Unpinning and Photovoltaic Model for P3HT:PCBM Organic Bulk Heterojunctions under Illumination. Chem. Phys. Lett. 2008, 465, 57-62.

(29) Yang, B.; Guo, F.; Yuan, Y.; Xiao, Z.; Lu, Y.; Dong, Q.; Huang, J. Solution-Processed Fullerene-Based Organic Schottky Junction Devices for Large-Open-Circuit-Voltage Organic Solar Cells. Adv. Mater. 2013, 25, 572-577.

(30) Seki, K. Energy Level Alignment and Interfacial Electronic Structures at Organic/Metal and Organic/Organic Interfaces. Adv. Mater. 1999, 11, 605-625.

(31) Osterloh, F. E.; Holmes, M. A.; Chang, L.; Moule, A. J.; Zhao, J. Photochemical Charge Separation in Poly(3-hexylthiophene) (P3HT) Films Observed With Surface Photovoltage Spectroscopy. J.Phys.Chem. C 2013, 117, 26905-26913.

(32) Kronik, L.; Shapira, Y. Surface Photovoltage Phenomena: Theory, Experiment, and Applications. Surf. Sci. Rep 1999, 37, 1-206.

(33) Kronik, L.; Shapira, Y. Surface Photovoltage Spectroscopy of Semiconductor Structures: At the Crossroads of Physics, Chemistry and Electrical Engineering. Surf. Interface Anal. 2001, 31, 954-965.

(34) Zhao, J.; Osterloh, F. E. Photochemical Charge Separation in Nanocrystal Photocatalyst Films-Insights from Surface Photovoltage Spectroscopy. J. Phys. Chem. Lett. 2014, 5, 782-786.

(35) Luria, J. L.; Hoepker, N.; Bruce, R.; Jacobs, A. R.; Groves, C.; Marohn, J. A. Spectroscopic Imaging of Photopotentials and Photoinduced Potential Fluctuations in a Bulk Heterojunction Solar Cell Film. ACS Nano 2012, 6, 9392-9401.

(36) Burstein, L.; Bregman, J.; Shapira, Y. Characterization of Interface States at III-V Compound Semiconductor-Metal Interfaces. J. Appl. Phys. 1991, 69, 2312-2316.

(37) Lagowski, J.; Jastrzebski, L.; Cullen, G. W. Electronic Characterization of Hetero-Epitaxial Silicon-on-Sapphire by Surface Photo-Voltage Spectroscopy. J. Electrochem. Soc. 1981, 128, 26652670.

(38) Musser, M. E.; Dahlberg, S. C. The Surface Photo-Voltage of Polymethine Semiconducting-Films. J. Chem. Phys. 1980, 72, 40844088.

(39) Moons, E.; Eschle, M.; Gratzel, M. Determination of the Energy Diagram of the Dithioketopyrrolopyrrole $/ \mathrm{SnO}_{2}: \mathrm{F}$ Heterojunction by Surface Photovoltage Spectroscopy. Appl. Phys. Lett. 1997, 71, 33053307.
(40) Li, Y.; He, Y. Fullerene Derivative Acceptors for High Performance OPV. Phys. Chem. Chem. Phys. 2011, 13, 1970-1983.

(41) Kang, H.; Yoon, S. C. Controlling Number of Indene Solubilizing Groups in Multiadduct Fullerenes for Tuning Optoelectronic Properties and Open-Circuit Voltage in Organic Solar Cells. ACS Appl. Mater. Interfaces 2012, 4, 110-116.

(42) Halls, J. J. M.; Walsh, C. A.; Greenham, N. C.; Marseglia, E. A.; Friend, R. H.; Moratti, S. C.; Holmes, A. B. Efficient Photodiodes from Interpenetrating Polymer Networks. Nature 1995, 376, 498-500.

(43) Heeger, A. J.; Yu, G. Polymer PV Cells: Enhanced Efficiencies via a Network of Internal Donor-Acceptor Heterojunctions. Science 1995, 270, 1789-1791.

(44) Chang, L.; Holmes, M. A.; Waller, M.; Osterloh, F. E.; Moule, A. J. Calcium Niobate Nanosheets as Novel Electron Transport Material for Solution-Processed Multijunction Polymer Solar Cell. J. Mater. Chem. 2012, 22, 20443-20450.

(45) Jacobson, A. J.; Johnson, J. W.; Lewandowski, J. T. Interlayer Chemistry between Thick Transition-Metal Oxide Layers: Synthesis and Intercalation Reactions of $\mathrm{K}\left[\mathrm{Ca} 2 \mathrm{Na}_{n-3} \mathrm{Nb}_{n} \mathrm{O}_{3 n+1}\right](3 \leq n \leq 7)$. Inorg. Chem. 1985, 24, 3727-3729.

(46) Schaak, R. E.; Mallouk, T. E. Self-Assembly of Tiled Perovskite Monolayer and Multilayer Thin Films. Chem. Mater. 2000, 12, 25132516.

(47) Wang, D. H.; Im, S. H.; Lee, H. K.; Park, O. O.; Park, J. H. Enhanced High-Temperature Long-Term Stability of Polymer Solar Cells with a Thermally Stable $\mathrm{TiO}_{x}$ Interlayer. J. Phys. Chem. C 2009, 113, 17268-17273.

(48) Roehling, J. D.; Arslan, I.; Moule, A. J. Controlling Microstructure in Poly(3-hexylthiophene) Nanofibers. J. Mater. Chem. 2012, 22, 2498-2506.

(49) Cook, S.; Ohkita, H.; Kim, Y.; Benson-Smith, J. J.; Bradley, D. D. C.; Durrant, J. R. A Photophysical Study of PCBM Thin Films. Chem. Phys. Lett. 2007, 445, 276-280.

(50) Kratschmer, W.; Lamb, L. D.; Fostiropoulos, K.; Huffman, D. R Solid C-60-A New Form Of Carbon. Nature 1990, 347, 354-358.

(51) Kazaoui, S.; Ross, R.; Minami, N. Intermolecular ChargeTransfer Excitation in C60 Films: Evidence from Luminescence and Photoconductivity. Phys. Rev. B 1995, 52, 11665-11668.

(52) Eilmes, A.; Munn, R. W.; Pac, B.; Petelenz, P. Charge-Transfer States and the Band Gap in Crystalline Fullerene. Chem. Phys. 1997, 214, 341-349.

(53) Munn, R. W.; Pac, B.; Petelenz, P. Charge-Transfer-Induced Frenkel Exciton Splitting in Crystalline Fullerene. Phys. Rev. B 1998, 57, 1328-1331.

(54) Tamura, H.; Tsukada, M. Role of Intermolecular Charge Delocalization on Electron Transport in Fullerene Aggregates. Phys. Rev. B 2012, 85, 054301.

(55) Haddon, R. C.; Brus, L. E. Electronic Structure and Bonding in C60. Chem. Phys. Lett. 1986, 125, 459-464.

(56) Leach, S.; Vervloet, M.; Despres, A.; Breheret, E.; Hare, J. P.; Dennis, T. J.; Kroto, H. W.; Taylor, R.; Walton, D. R. M. Electronic Spectra and Transitions of the Fullerene C60. Chem. Phys. 1992, 160, 451-466.

(57) Mishori, B.; Shapira, Y.; BeluMarian, A.; Manciu, M.; Devenyi, A. Studies of C-60 Thin Films Using Surface Photovoltage Spectroscopy. Chem. Phys. Lett. 1997, 264, 163-167.

(58) Bisquert, J.; Garcia-Belmonte, G.; Munar, A.; Sessolo, M.; Soriano, A.; Bolink, H. J. Band Unpinning and Photovoltaic Model for P3HT:PCBM Organic Bulk Heterojunctions Under Illumination. Chem. Phys. Lett. 2008, 465, 57-62.

(59) Sirringhaus, H.; Brown, P. J.; Friend, R. H.; Nielsen, M. M.; Bechgaard, K.; Langeveld-Voss, B. M. W.; Spiering, A. J. H.; Janssen, R. A. J.; Meijer, E. W.; Herwig, P.; et al. Two-Dimensional Charge Transport in Self-Organized, High-Mobility Conjugated Polymers. Nature 1999, 401, 685-688.

(60) Xu, Z.; Chen, L. M.; Chen, M. H.; Li, G.; Yang, Y. Energy Level Alignment of Poly(3-hexylthiophene): 6,6-Phenyl C-61 Butyric Acid Methyl Ester Bulk Heterojunction. Appl. Phys. Lett. 2009, 95, 013301. 
(61) Davis, R. J.; Lloyd, M. T.; Ferreira, S. R.; Bruzek, M. J.; Watkins, S. E.; Lindell, L.; Sehati, P.; Fahlman, M.; Anthony, J. E.; Hsu, J. W. P. Determination of Energy Level Alignment at Interfaces of Hybrid and Organic Solar Cells Under Ambient Environment. J. Mater. Chem. 2011, 21, 1721-1729.

(62) Roehling, J. D.; Batenburg, K. J.; Swain, F. B.; Moulé, A. J.; Arslan, I. Three-Dimensional Concentration Mapping of Organic Blends. Adv. Funct. Mater. 2013, 23, 2115-2122.

(63) Wodo, O.; Roehling, J. D.; Moule, A. J.; Ganapathysubramanian, B. Quantifying Organic Solar Cell Morphology: A Computational Study of Three-Dimensional Maps. Energy Environ. Sci. 2013, 6, 30603070.

(64) Mauger, S. A.; Chang, L.; Friedrich, S.; Rochester, C. W.; Huang, D. M.; Wang, P.; Moulé, A. J. Self-Assembly of Selective Interfaces in Organic Photovoltaics. Adv. Funct. Mater. 2013, 23, 1935-1946.

(65) Fefer, E.; Kronik, L.; Leibovitch, M.; Shapira, Y.; Riedl, W. InSitu Monitoring of Surface Chemistry and Charge Transfer at Semiconductor Surfaces. Appl. Surf. Sci. 1996, 104, 61-67.

(66) Ishii, H.; Sugiyama, K.; Ito, E.; Seki, K. Energy Level Alignment and Interfacial Electronic Structures at Organic Metal and Organic Organic Interfaces. Adv. Mater. 1999, 11, 605-625.

(67) Würfel, P. Physics of Solar Cells From Principles to New Concepts; Wiley-VCH: Weinheim, 2005; p 244.

(68) Nelson, J. The Physics of Solar Cells; Imperial College Press: London, 2004; p 363.

(69) Glatthar, M.; Mingirulli, N.; Zimmermann, B.; Ziegler, T.; Kern, R.; Niggemann, M.; Hinsch, A.; Gombert, A. Impedance Spectroscopy on Organic Bulk-Heterojunction Solar Cells. Phys. Status Solidi A 2005, 202, R125-R127.

(70) Garcia-Belmonte, G.; Munar, A.; Barea, E. M.; Bisquert, J.; Ugarte, I.; Pacios, R. Charge Carrier Mobility and Lifetime of Organic Bulk Heterojunctions Analyzed by Impedance Spectroscopy. Org. Electron. 2008, 9, 847-851.

(71) Di Nuzzo, D.; van Reenen, S.; Janssen, R. A. J.; Kemerink, M.; Meskers, S. C. J. Evidence for Space-Charge-Limited Conduction in Organic Photovoltaic Cells at Open-Circuit Conditions. Phys. Rev. B 2013, 87, 085207.

(72) Morfa, A. J.; Nardes, A. M.; Shaheen, S. E.; Kopidakis, N.; van de Lagemaat, J. Time-of-Flight Studies of Electron-Collection Kinetics in Polymer:Fullerene Bulk-Heterojunction Solar Cells. Adv. Funct. Mater. 2011, 21, 2580-2586.

(73) Tengstedt, C.; Osikowicz, W.; Parker, I. D.; Hsu, C. H.; Fahlman, M. Fermi-Level Pinning at Conjugated Polymer Interfaces. Appl. Phys. Lett. 2006, 88, 053502.

(74) Xu, Z.; Chen, L. M.; Chen, M. H.; Li, G.; Yang, Y. Energy Level Alignment of Poly-3-hexylthiophene: 6,6-Phenyl C61 Butyric Acid Methyl Ester Bulk Heterojunction. Appl. Phys. Lett. 2009, 95, 013301.

(75) Lide, D. R, Electron Work Function of the Elements. CRC Handbook of Chemistry and Physics; CRC Press: Boca Raton, FL, 2008.

(76) Xu, Y.; Schoonen, M. A. A. The Absolute Energy Positions of Conduction and Valence Bands of Selected Semiconducting Minerals. Am. Mineral. 2000, 85, 543-556.

(77) Tvrdy, K.; Frantsuzov, P. A.; Kamat, P. V. Photoinduced Electron Transfer From Semiconductor Quantum Dots to Metal Oxide Nanoparticles. Proc. Natl. Acad. Sci. U.S.A. 2011, 108, 29-34.

(78) Hamilton, R.; Shuttle, C. G.; O’Regan, B.; Hammant, T. C.; Nelson, J.; Durrant, J. R. Recombination in Annealed and Nonannealed Polythiophene/Fullerene Solar Cells: Transient Photovoltage Studies versus Numerical Modeling. J. Phys. Chem. Lett. 2010, 1, 1432-1436.

(79) Padinger, F.; Rittgerber, R. S.; Sariciftci, S. Effects of Postproduction Treatment on Plastic Solar Cells. Advanced Functional Materials. Adv. Funct. Mater. 2003, 13, 85.

(80) Moule, A. J.; Meerholz, K. Morphology Control in SolutionProcessed Bulk-Heterojunction Solar Cell Mixtures. Adv. Funct. Mater. 2009, 19, 3028-3036. 